\title{
Trivium
}

Revue franco-allemande de sciences humaines et sociales - Deutsch-französische Zeitschrift für Geistesund Sozialwissenschaften

$6 \mid 2010$

Esthétique et science de l'art

\section{Was ist Ästhetik? [1878]}

\section{Eugène Véron}

Traducteur : Mira Köller

\section{Q OpenEdition}

\section{Journals}

\section{Édition électronique}

URL : http://journals.openedition.org/trivium/3658

DOI : $10.4000 /$ trivium.3658

ISSN : 1963-1820

\section{Éditeur}

Les éditions de la Maison des sciences de l'Homme

\section{Référence électronique}

Eugène Véron, « Was ist Ästhetik? [1878] », Trivium [Online], 6 | 2010, online erschienen am 05 Mai 2010, abgerufen am 10 Dezember 2020. URL : http://journals.openedition.org/trivium/3658 ; DOI : https://doi.org/10.4000/trivium.3658

Ce document a été généré automatiquement le 10 décembre 2020.

\section{(c) (i) () $\Theta$}

Les contenus des la revue Trivium sont mis à disposition selon les termes de la Licence Creative Commons Attribution - Pas d'Utilisation Commerciale - Pas de Modification 4.0 International. 


\title{
Was ist Ästhetik? [1878]
}

\author{
Eugène Véron
}

Traduction : Mira Köller

\section{§ 1. Das Schöne - die Unzulänglichkeit dieses Prinzips, um die Kunst zu erklären - die Nachahmungstheorie ist nicht viel akzeptabeler - Definition}

1 Wir haben gerade die Kunst definiert, nun müssen wir erklären, was wir unter dem Wort Ästhetik verstehen. Definieren Sie die Wörter, sagte Voltaire, der, nachdem er sein Leben damit verbracht hatte, Polemiken aller Art zu führen, aus persönlicher Erfahrung wusste, dass es eine ernsthafte Diskussion nur unter der Voraussetzung gibt, dass man sich zuvor über die genaue Bedeutung der von beiden Seiten gebrauchten Wörter geeinigt hat.

Diese auf jeden Fall richtige Vorsichtsmaßnahme ist vor allem notwendig für das, was die von den Metaphysikern verkomplizierten Fragen berührt. Von den Fragen, die sie am erfolgreichsten verdunkelt haben, kann man jene, die sich auf die Ästhetik beziehen, an die erste Stelle setzen.

Was ist Ästhetik?

4 Etymologisch kommt dieser Begriff von einem griechischen Wort, das Empfindung, Wahrnehmung bedeutet. Die Ästhetik wäre demnach die Wissenschaft, die sich mit Empfindungen oder Wahrnehmungen beschäftigt. Mit allen im Allgemeinen oder mit einigen im Besonderen? Aus dem Wort geht das nicht hervor.

Im ersten Fall wäre es eine vollständige Philosophie, denn es gibt keine menschliche Tatsache, die, philosophisch gesprochen, nicht auf eine Empfindung oder eine Wahrnehmung zurückgeführt werden kann. Im zweiten Fall ist das Wort nicht präzise genug, denn nichts zeigt an, um welche Empfindungen oder Wahrnehmungen es sich handelt. Das Wort ist also schlecht konzipiert. Doch da es in den Sprachgebrauch eingegangen ist, werden wir es mangels eines besseren behalten. 
Die Ästhetik wird als die Wissenschaft vom Schönen definiert, was auf den ersten Blick befriedigender erscheint, vor allem für diejenigen, die glauben, die Kunst insgesamt zu definieren, wenn sie sie als Suche nach dem Schönen bezeichnen; aber wenn man nur ein wenig nachdenkt, wird einem schnell gewahr, dass es dieser Definition gut täte, selbst definiert zu werden.

Die Wissenschaft vom Schönen. Also gut! Aber was ist das Schöne?

Dieses abstrakte Wort sieht nach einer platonischen Wesenheit aus, die uns zunächst einmal misstrauisch macht, wie alles, was im Gewand der Metaphysik daherkommt. Von der Antike bis heute haben fast alle ästhetischen Lehren, die von der Auffassung des Schönen ausgehen, dieses als etwas Göttliches, Absolutes betrachtet, das seine distinkte Wirklichkeit außerhalb des Menschen hat. Die wenigen Metaphysiker, die einen anderen Standpunkt eingenommen haben, haben nur einen sehr begrenzten Einfluss auf die Kunst ausgeübt. Wir lassen sie beiseite. Für Platon wie für Winckelmann, und auch heute noch für die akademische Schule, ist das Schöne, an sich betrachtet, da es eines der Attribute der göttlichen Vollkommenheit ist, wie alles Absolute »eins und nicht verschieden « und folglich einzig und universell; es setzt sich durch, unwandelbar, zu allen Zeiten, bei allen Rassen, in allen Künsten. In seiner Anwendung ist das Schöne die wesentliche Form aller mannigfaltigen Schöpfungen, bevor sie Gestalt angenommen haben; das heißt, es ist der Prototyp der Schöpfung, so wie sie in ihrer vollkommenen Form im Gehirn des Schöpfergottes erschienen sein musste, bevor sie die Herabstufung erfährt, die die notwendige Folge ihrer Verwirklichung in der Materie ist.

9 Von dem Augenblick an, da man einen derart beschaffenen Geist besitzt, dass man die Schönheit der Formen außerhalb der materiellen Wirklichkeit denken kann, ist die Bestimmung des metaphysischen und folglich des einen, allgemeingültigen und unveränderlichen Schönen nur noch eine Frage der Logik. Der Ausgangspunkt mag absurd sein, aber das hat die Metaphysiker noch nie gestört, wenn die Konsequenz nur ordnungsgemäß von gültigen Syllogismen abgeleitet ist.

10 Aus demselben Grund wird das solchermaßen aufgefasste Schöne natürlicherweise zum höchsten und einzigen Modell aller Künste, zum ewigen Vorbild der Anstrengungen aller Menschen, zum Ziel all ihres Strebens. Unter diesem Gesichtspunkt betrachtet heißt es Ideal und ist nichts anderes als der blasse Abglanz des Wahren ${ }^{1}$, das nur in der Welt der intelligiblen und reinen Ideen existiert.

11 Diese Auffassung des Schönen ist sicherlich die am weitesten verbreitete. Sie wird auf allen Stufen des Universitätsstudiums propagiert und beherrscht dadurch die öffentliche Auffassung.

Im Grunde beruht sie auf einer reinen Hypothese, die durch absolut nichts begründet ist und die, wie alle gleichartigen metaphysischen Wesenheiten, nur durch die verbale Realität des Wortes, kraft deren es entstanden ist, äußeren Schein gewinnt. Richtig ist, dass die Sprache, sei es zunächst aus Unfähigkeit, die Empfindungen zu analysieren und zwischen ähnlichen Wahrnehmungen zu unterscheiden, sei es später aus dem Bedürfnis heraus, zu vereinfachen und zu verallgemeinern, im Ausdruck >Schönheit die Gesamtheit der bewundernden Ausdrücke zusammengefasst hat; aber das genügt nicht, um den Metaphysikern das Recht zu geben, auf die wesentliche und substantielle Einheit der Ursache dieser in Wirklichkeit so verschiedenartigen Eindrücke zu schließen. 
13 Es ist völlig unmöglich, dass diese Auffassung des Schönen dem Streben der Künstler genügt, es sei denn, aus dem Bereich der Kunst wird ein Gutteil der Werke ausgeschlossen, die dem menschlichen Genie zur größten Ehre gereichen, oder dem Wortsinn wird Gewalt angetan. In Wirklichkeit wendet sich die Kunst ausnahmslos an alle Gefühle: Hoffnung oder Schrecken, Schmerz oder Freude, Hass oder Liebe; sie gibt alle Emotionen wieder, die das Herz des Menschen bewegen, ohne sich um ihren Bezug zur sichtbaren oder zur idealen Vollkommenheit zu kümmern. Sie drückt sogar das Hässliche und Schreckliche aus, ohne deshalb aufzuhören, Kunst zu sein und Bewunderung $\mathrm{zu}$ verdienen. Haben das Schlachtfeld von Preußisch-Eylau, die furchtbaren oder grauenhaften Martern der Verdammten, die Verbrechen und Schandtaten der grausamen Bestien, die unter dem Namen Cäsar die römische Welt in Angst und Schrecken versetzten, Gros, Dante, Tacitus nicht den Anlass zur Schaffung großartiger Werke geboten, deren Modell in der Welt des Intelligiblen nur schwer vorzufinden wäre? Welche Schönheit kann man auf einem mit Toten und Sterbenden übersäten Schlachtfeld suchen? Was ist schön am Anblick des Grafen Ugolin, der das Fleisch seiner eigenen Kinder verschlingt, oder an dem von Tiberius auf der Insel Capri?

14 Beispiele dieser Art finden sich überall, in allen Künsten. Die klassischsten Gedichte sind voll davon. Schon gleich am Anfang der Ilias beschimpfen sich Achill und Agamemnon gegenseitig mit einem Eifer und in einem Stil, dem die kühnsten Realisten unserer Tage nicht die Anerkennung versagen würden. Die um Patroklos' Grabstätte herumgeschleifte Leiche Hektors, das Porträt von Thersites, all diese Szenen von ununterbrochen aufeinander folgenden Massakern, Ödipus, der sich die Augen aussticht und blutüberströmt erscheint, laut seinen Schmerz kundzutun, Herkules, der seine Kinder in einem Anfall von rasendem Wahnsinn hinmordet, und Medea, die ihren Kindern die Kehle durchschneidet, um sich für eine Rivalin zu rächen, die Furien, die Orest verfolgen, und tausend andere ähnliche Stücke liefern reichlich Belege dafür, dass die Griechen selbst, trotz Platon, den Bereich der Kunst nicht auf die Suche nach dem Schönen beschränkt haben.

15 Was kann man Schönes finden an den mehr oder weniger Abscheu erregenden oder schändlichen Lastern der ungeheuer vielen bejammernswerten Gestalten, die zu allen Zeiten und in allen Ländern die Literatur bevölkern? Wo ist die Schönheit bei Nero, bei Agrippina, bei Madame Bovary oder Valérie Marneffe? Wie kommt es, dass die Schilderung jener Feigheiten und Schändlichkeiten, die uns in der Wirklichkeit entsetzen, in den Kunstwerken eine völlig entgegengesetzte Wirkung erzielen können?

Erklärt wird diese Sonderbarkeit mit einer natürlichen Wirkung der Nachahmung. Boileau, der kaum des Realismus verdächtigt werden kann, konnte ohne Proteste auszulösen sagen:

Es gibt keine Schlange oder abscheuliches Ungeheuer,

das, von der Kunst nachgeahmt, dem Auge nicht gefallen würde.

Vor ihm hatte Aristoteles gesagt: "[...] alle Menschen haben Freude an der Nachahmung. Beweis dafür ist die Erfahrung, die wir angesichts ihrer Werke an uns machen. Denn auch bei Dingen, die wir in Wirklichkeit nur mit Widerwillen betrachten, sehen wir ihre sorgfältige bildliche Wiedergabe mit Freude, z.B. Bilder von häßlichen Tieren und von Toten. ${ }^{2}$ Pascal, obwohl er einen anderen Standpunkt vertritt, stellt dasselbe fest: »Wie eitel ist doch die Malerei, welche zur Bewunderung reizt, indem sie Abbilder von dem schafft, was man nicht einmal im Original bewundert! « ${ }^{3}$ 
Diese Erklärung zieht die Verneinung der Theorie, die aus dem Schönen ein Abbild der Vollkommenheit macht, nach sich. Sie impliziert gleichzeitig eine Zweiteilung der Frage und zwingt $\mathrm{zu}$ einer grundsätzlichen Unterscheidung zwischen dem Naturschönen und dem Kunstschönen. Nur das erste würde in Verbindung mit der Idee der Vollkommenheit bleiben, das zweite ginge aus einer rein akzidentellen und menschlichen Tatsache hervor, der Nachahmung. Wir werden noch auf diese Unterscheidung zurückkommen und ihre Geltung beurteilen müssen. Im Augenblick befassen wir uns nur mit dem Kunstschönen.

Stimmt es denn, dass ein in der Wirklichkeit schrecklich anzusehender Anblick allein dadurch schön wird, dass er nachgeahmt wird? Ist es wirklich die Ähnlichkeit mit dem Gegenstand, die die Schönheit des Kunstwerks erzeugt? Mitnichten. Aristoteles, Boileau, Pascal sowie alle Anhänger der Nachahmungstheorie lassen sich von einem Schein blenden, der einer Untersuchung nicht standhält.

19 Nehmen Sie den geschicktesten Künstler und bitten Sie ihn um ein Porträt von Thersites oder von Quasimodo. Diese schrecklichen Gestalten bleiben deshalb als Gestalten nicht weniger schrecklich, und der Künstler wird darüber nicht hinwegtäuschen. Das Porträt eines hässlichen Menschen bleibt hässlich, wenn die Darstellung originalgetreu ist, so wie auch die genaue Abbildung der Züge von Antinous oder Adonis uns unvermeidlich das Gefühl einer schönen Gestalt vermitteln wird.

Aber gleichzeitig kann es durchaus geschehen, dass das Porträt von Quasimodo, wie hässlich es auch immer sein mag, dem des Antinous, weise dieses auch noch so viel Ähnlichkeit auf, als Kunstwerk unendlich überlegen ist - eine Tatsache, die denen entgangen ist, die sich einbilden, dass die Nachahmung das höchste Ziel der Kunst sei, und die Genauigkeit der unfehlbare Maßstab für den Wert des Werks.

21 Diese Tatsache ist nun sehr wichtig, erlaubt sie uns doch, das eigentliche Wesen der Kunst $\mathrm{zu}$ begreifen und $\mathrm{zu}$ verstehen, warum sie eine so hohe Stellung unter den Äußerungen des Genius des Menschen hat.

22 Zunächst einmal: Wenn jegliche Anstrengung des Künstlers sich auf die Nachahmung von Gegenständen beschränken sollte, so kämen wir unweigerlich zu dem Schluss, dass die Kunst ihre Rolle schon jetzt ausgespielt hat, zumindest was die Wiedergabe von Formen und Linien betrifft, da unter diesem Gesichtspunkt vernünftigerweise keine Nachahmung eine größere Genauigkeit beanspruchen kann als die der Fotografie. Wenn die Malerei überhaupt noch einen Daseinsgrund hat, dann deshalb, weil sie gegenüber der Maschine noch immer den Vorteil besitzt, die Farbe wiederzugeben. Aber wenn es der Chemie gelingt, was wahrscheinlich ist, diesen letzten Schritt zu vollziehen, dann wird die Kunst, da sie keine ihr eigene Funktion mehr hat, den Platz ganz räumen müssen, ebenso wie in der Industrie die mechanische Arbeit jeden Tag darauf abzielt, die Handarbeit immer weiter zu verdrängen.

Die Genauigkeit der Nachahmung kann, dies geben wir durchaus zu, in gewisser Hinsicht ihre Nützlichkeit und ihre Bedeutung haben, wenn es sich zum Beispiel um das Porträt eines berühmtes Mannes handelt, der einen herausragenden Platz in der Geschichte einnimmt, um die Schilderung einer Leidenschaft, eines Charakters. Hier bedarf es der Ähnlichkeit und der Genauigkeit im Detail. Die Porträts von Richelieu, von Ludwig XIV., von Napoleon sind für uns historische Zeugnisse. Wir dulden nicht, dass ein Dichter oder ein Maler sie für uns in Zügen darstellt, die nicht mit der Wirklichkeit ihrer historischen Rolle übereinstimmen. Warum interessieren uns die 
psychologischen Beschreibungen von La Bruyère, von Molière, von Balzac so sehr? Ist es nicht zumindest teilweise deshalb, weil sie wahr sind und uns ermöglichen, im Gefolge dieser großen Geister in jene Geheimnisse des menschlichen Herzens einzudringen, die wir ohne sie weniger gut kennen würden?

Man darf allerdings die Bedeutung dieser Treue der Nachahmung nicht überbewerten. Zumindest ist eine wesentliche Unterscheidung vorzunehmen. Der Historiker und der Moralist müssen natürlich gerade der Genauigkeit der Nachbildung große Wichtigkeit beimessen. Von ihrem besonderen Standpunkt aus gesehen ist es von außerordentlich großem Nutzen, in den historischen Porträts den eigentlichen Charakter der Männer wiederzufinden, die einen großen Einfluss auf das Schicksal ihrer Mitmenschen gehabt haben, und in den psychologischen Beschreibungen die Züge, die ihnen helfen, die Leidenschaften, die kleinen Schwächen und Laster der Menschheit zu verstehen und zu erklären. Sie müssen sich auf sicherem Gelände fühlen, und sie sind den Malern, den Dichtern, den Beobachtern dankbar, die ihnen den Dienst erweisen, ihre Forschungen zu erleichtern.

Aber vom ästhetischen Standpunkt aus betrachtet, dem einzigen, mit dem wir uns hier zu beschäftigen haben, kann der Wert eines Werkes nicht nach der Zahl der Dienste beurteilt werden, die es zu leisten vermag. Dieses Kriterium ist das der Wissenschaft und der Industrie, nicht das der Kunst.

Nehmen Sie zum Beispiel einmal die Porträts, die in den Memoiren von Saint Simon mit so erstaunlicher Kraft und so großer Anschaulichkeit brillant hingeworfen sind. Worin besteht ihr Wert? Ähnlich zu sein? Das vermögen wir kaum zu beurteilen, da wir die Modelle nicht vor Augen haben. Nein, uns begeistert der leidenschaftliche Schwung dieses Mannes, die Konzentrationskraft, mit der er die wesentlichen Züge einer Physiognomie erfasst und in wenigen Worten wiedergibt, das Feuer der Leidenschaft, mit dem er dem Hass oder der Verachtung freien Lauf lässt, die die meisten Originale in ihm erregen, deren Silhouette zu skizzieren er sich die Mühe macht, wie um das Schlechte zu rechtfertigen, das er über sie sagt. Es ist wenig wahrscheinlich, dass er mit einem solchen Charakter zu der Unparteilichkeit fähig ist, deren es für solche Beschreibungen bedarf, die den Anspruch erheben, absolut historische Zeugnisse zu sein; und solange dies nicht anderweitig bewiesen würde, dürfte man durchaus annehmen, dass er keine Hemmungen gehabt hat, einige Züge boshaft herauszustreichen und andere im Dunkeln zu belassen.

Und doch bilden diese Memoiren aus ästhetischer Sicht eine erstklassige Galerie, weil alle diese Darstellungen statt Ähnlichkeit Bewegung und Leben haben. Man spürt, dass der Autor sie originalgetreu abzubilden glaubte und dies auch wollte, dass er alle seine Gestalten so gezeichnet hat, wie er sie wirklich in seiner Leidenschaft gesehen hat. In der Kunst ersetzt die Aufrichtigkeit die Wahrheit.

Kurz, der Grad der Wirklichkeitswiedergabe, den ein Kunstwerk besitzt, hat ästhetische Bedeutung nur, weil er uns erlaubt, die Verstandesschärfe zu ermessen, deren es bedurfte, um sie zu erfassen, sowie die Vorstellungskraft, die es ermöglichte, sie mit dieser von uns bewunderten Anschaulichkeit wiederzugeben.

Die Bedingungen sind dieselben, wenn es sich statt um das Porträt eines Individuums um die Beschreibung einer Leidenschaft, eines Charakters handelt. Ästhetischen Wert gewinnen die Genauigkeit und die Tiefe der Züge allein dadurch, dass sie vom Arbeitseifer des Künstlers zeugen, von seinem Bewusstsein, seiner Verstandesschärfe, seiner Emotion und von seinem Vermögen, seinen Eindruck in Worte zu kleiden. Die 
Nachahmung ist lediglich Mittel oder vielmehr Anlass und Vorwand. Die wahre, die einzige Quelle der Kunst ist immer der Künstler. In diesem Fall, wie im anderen, ist die dem Modell innewohnende Schönheit von völlig zweitrangiger Bedeutung.

\section{§ 2. Wir bewundern im Kunstwerk das Genie des Künstlers - Definition der Ästhetik}

30 Wenn wir die Entwicklung der Charaktere von Tartuffe, dem Geizigen, Cousine Bette oder der Marneffe miterleben, interessiert uns, ästhetisch gesprochen, weder Tartuffe noch Harpagon, noch die Marneffe, noch Cousine Bette, sondern die Tiefe der Beobachtung, dank der Molière und Balzac bis ins Innerste dieser Charaktere vordringen konnten, vor allem die beschwörende Kraft, mit der sie die Charaktere aus dieser Beobachtung hervortreten lassen, um sie auf der Bühne und im Roman zur Schau zu stellen und aus ihnen lebendige Wesen zu machen. An ihnen bewundern wir nicht sie selbst, sondern das Genie, das sie geschaffen und in Bewegung versetzt hat, das sie ein so einzigartiges und intensives Leben leben lässt, dass sie, einmal dem Gedächtnis eingeprägt, nicht mehr daraus $\mathrm{zu}$ streichen sind und wie eine unauslöschliche Vision in der Erinnerung haften bleiben. Wenn wir sie sprechen und handeln sehen, sowohl auf den Seiten des Buches als auch auf den Brettern der Bühne, bleiben wir hingerissen von dieser außerordentlichen Magie, diesem Wunder an Intuition, das es den Schöpfern ermöglicht hat, die Wahrnehmungen ihres Gehirns für alle sichtbar und wirklich zu machen, aus ihnen vollständige Bilder zu entwickeln, die lebendiger sind als ihre Modelle, diese Gespenster mit einer inneren und sich ausbreitenden Schwingung $\mathrm{zu}$ beseelen, die die wirklichen Lebewesen nicht in derselben Stärke besitzen und die sie geradewegs in die höhere Welt eintreten lässt, in der die von der menschlichen Einbildungskraft geschaffenen, unsterblichen Typen leben. Ohne aufzuhören, wahr zu sein, gehen sie über die Wirklichkeit hinaus, der sie entspringen; sie verdichten und vervollständigen sie durch ihre wirklich bedeutsamen Züge, befreit von Nebensächlichkeiten, die die klare Sicht trüben, und mittels dieser Verdichtung erreichen sie eine Intensität der Wirkung, die in der Natur nicht anzutreffen ist. Darin beruht der Beitrag der Kunst, und dank ihm werden diese Schöpfungen dann ihrerseits zu Modellen.

31 Was stellen diese Typen nun aber für uns dar? Die Scheinheiligkeit, den Geiz, den Neid, die Prostitution. Wird man sagen, dass die Schönheit, die wir in der Darstellung dieser scheußlichen Laster wiederfinden, in ihnen selbst existiert? Natürlich nicht, sie liegt ganz in der Kunst, in der Persönlichkeit der Dichter, deren Vermögen diese lebensvollen Bilder geschaffen hat. Uns beeindruckt also nicht nur die Genauigkeit der Nachahmung, sondern vor allem die Kunst, die aus dem von der Wirklichkeit gelieferten Material diese vollkommenen Gebilde gewonnen hat. Wir bewundern nicht die dargestellten Laster, sondern das Genie der Menschen, die sie so wunderbar verstanden und dargestellt haben; kurz, nicht die Originale erscheinen uns schön, sondern ihr Porträt, und das aus demselben Grund, aus dem das Porträt eines Quasimodo ein schönes Kunstwerk sein kann.

Um andere Beispiele $\mathrm{zu}$ nennen: Was beeindruckt uns am Freskogemälde der Sixtinischen Kapelle, wo Michelangelo für uns die Scheidung von Licht und Finsternis darstellt? Natürlich hat das gar nichts mit Nachahmung zu tun. Niemand, Michelangelo so wenig wie irgendwer sonst, war bei der Erschaffung des Lichts dabei. Die 
Einbildungskraft des Künstlers konnte sich also völlig frei entfalten. Der Wert seines Werkes war ganz der Kraft untergeordnet, mit der er die Idee wiedergeben würde, die er sich von einem Schauspiel machte, dessen einzelne Teile er nur in sich selbst finden konnte.

Selbst die Bibel konnte ihm hinsichtlich der Nachahmung nicht hilfreich sein. Jahve sprach: »Es werde Licht!, und es ward Licht.« Wie sollte die Energie des schöpferischen Wortes mit den Mitteln der Malerei dargestellt werden? Daran war gar nicht zu denken. Nun verfügt der Maler über ganz andere Mittel als der Dichter. Der eine richtet sich über die Ohren an den Geist, der andere über die Augen. Genau das hat Michelangelo verstanden. So hat er das Wort durch die Geste ersetzt, und es ist ihm nicht weniger gut gelungen als dem Autor der Genesis ${ }^{4}$, den Eindruck von souveräner Größe und Macht wiederzugeben, den in seiner Einbildungskraft der Akt erzeugt hatte, den er darstellen wollte.

Wenn Ruisdael uns einen vom Wind gepeitschten Busch zeigt, interessiert uns dann die Individualität dieses Busches? ${ }^{5}$ Müssen wir, um ergriffen zu sein, sicher sein, dass der Busch, den er gemalt hat, ganz genau dem wirklichen Busch ähnlich ist, der ihm als Modell gedient hat? Was kümmert uns das? Uns genügt, dass es tatsächlich ein Busch ist, und uns berührt der Charakter, von dem dieses Werk geprägt ist und der uns genau den Eindruck seines Schöpfers vermittelt.

Wir könnten dasselbe von der Ilias, der Odyssee, den Tragödien von Aischylos, von Sophokles, von Euripides, von Corneille, den Dramen von Shakespeare und von Victor Hugo, von der Göttlichen Komödie, von allen großen Werken des menschlichen Genies sagen. Wem würde es bei ihrer Lektüre einfallen, sich zu fragen, ob sie mit der Realität der Tatsachen übereinstimmen?

Nachahmung ist nicht das Ziel der Kunst, wie es auch nicht das Ziel des Schriftstellers ist, Buchstaben und Silben zusammenzufügen, sondern mithilfe von daraus gebildeten Wörtern seine Gedanken und Gefühle auszudrücken. Wenn der Dichter seine Verse schreibt, wenn der Musiker seine Noten arrangiert, dann wissen sie genau, dass ihr wirkliches Ziel darüber hinausgeht.

Wie bereits erläutert, ist diese Unterscheidung bei der Malerei und der Skulptur weniger klar. Einige Künstler, und nicht die Ungeschicktesten, sind völlig davon überzeugt, dass sie, wenn sie dem Modell gegenüberstehen, dieses nur nachahmen. Und in der Tat machen sie nichts anderes, und durch diese Nachahmung gelingt es ihnen, Werke von unbestreitbar künstlerischem Wert zu schaffen.

Das ist ein schlichtes Missverständnis.

Wenn der Künstler sich wirklich auf die Rolle der Kopiermaschine beschränken könnte, wenn er so vollständig verschwinden könnte, dass sein Werk auf die sklavische Nachbildung aller Züge und aller Einzelheiten des vor seinen Augen präsentierten Gegenstands oder Schauspiels beschränkt wäre, dann hätte sein Werk ganz einfach nur den Wert eines mehr oder weniger genauen Protokolls und bliebe tatsächlich zwangsläufig hinter der Wirklichkeit zurück. Welcher Künstler würde es auf sich nehmen, eine echte Sonne zu malen, ohne ein wenig zu mogeln, ohne bestimmte Mittel zur Hilfe zu nehmen, zu denen die Sonne sich in der Wirklichkeit nicht herablässt? Es ist wissenschaftlich erwiesen, dass das Licht noch in den hellsten Gemälden im Vergleich zur wirklichen Helle des Tages wie eine Kerze zur Sonne ist. Es ist also ganz klar, dass der Maler die Empfindung des Lichts nur durch eine Reihe von Arrangements 
und Kunstgriffen erzeugen kann, die allein schon durch ihre Kunstfertigkeit das Werk zu etwas Persönlichem machen. Aber lassen wir das.

Gerade dadurch, dass er mit Feingefühl und Vorstellungskraft begabt ist, befindet sich der Künstler gegenüber den Gegenständen der Natur oder den Schauspielen der Geschichte in einer besonderen Situation, ob er es will oder nicht. Für wie realistisch auch immer er sich halten mag, seine Themen wählt er doch nicht zufällig. Nun zeigt aber allein schon die Wahl des Themas eine Präferenz, die aus einem mehr oder weniger bestimmten Eindruck und einer mehr oder weniger verborgenen Übereinstimmung zwischen dem Charakter des objekts und dem des Künstlers hervorgeht. Diesen Eindruck und diese Übereinstimmung wiederzugeben, darum geht es und dies ist das wirkliche Ziel seiner Anstrengung. Ob er sich dessen bewusst ist oder nicht, darin liegt der künstlerische Wert seines Werkes, und darin ist er Künstler. Ohne es zu wollen, selbst ohne es zu ahnen, interpretiert er die Natur und bringt ihre Züge auf den vorherrschenden Eindruck zurück, der ihm den Pinsel in die Hand nehmen ließ. Er drückt seinem Werk diesen akzidentellen Stempel auf, der dem des anhaltenden Genies, das seine Persönlichkeit ausmacht, hinzugefügt wird. Der Dichter, der Musiker, der Bildhauer, der Architekt: sie alle gehorchen mehr oder weniger demselben Gesetz. Darauf lassen sich alle künstlerischen Kompositionsregeln zurückführen, die die akademische Pedanterie bis zur Spitzfindigkeit, bis zum Widerspruch vermehrt hat.

41 Je ausgeprägter der persönliche Charakter in einem Kunstwerk ist, desto beeindruckender ist die Mitwirkung aller Einzelheiten beim Ausdruck des Ganzen, desto deutlicher kann man in jedem Teil das Gefühl von Größe, von Traurigkeit, von Freude erkennen, das der Künstler empfunden hat, desto offensichtlicher ist die Unterordnung der Nachahmung unter die rein menschliche Entscheidung für die vorherrschende Empfindung oder den vorherrschenden Willen, desto wahrscheinlicher ist es auch, dass das Ergebnis früher oder später einhellige Bewunderung hervorrufen wird, freilich unter der Voraussetzung, dass das ausgedrückte Gefühl in die Kategorie der allgemeinen Gefühle der Menschheit passt und dass die Ausführung nicht so geartet ist, dass sie die Kenner abstößt oder verwirrt. Es ist in der Tat nicht ausgeschlossen, dass ein mit einer gestörten oder krankhaften Phantasie begabter Künstler sich dadurch außerhalb der normalen Bedingungen begibt und sich solchermaßen dazu verdammt, vom Publikum nicht verstanden $\mathrm{zu}$ werden. $\mathrm{Zu}$ persönliche Eindrücke, zu exzentrische Empfindungen, Bizarrheiten in Ausführung und Verfahren können ihm, ohne dem inneren Wert der Inspiration oder des Werkes Abbruch zu tun, einen so sonderbaren und eigentümlichen Charakter verleihen, dass es unmöglich wird, seinen Wert herauszufiltern. Die Übertreibung der besten Qualitäten verwandeln sie in Mängel. Die Persönlichkeit, die aus der Imitation ein Kunstwerk macht, indem sie sich ihr hinzufügt, verwandelt es in ein Rätsel, wenn sie bis zur Absonderlichkeit getrieben wird.

42 Wenn es uns gelungen ist, uns verständlich zu machen, wird also sichtbar, dass das Kunstschöne im Wesentlichen durch das Wirken des menschlichen Genies entsteht, das durch eine besondere Empfindung mehr oder weniger angeregt wird. Das Werk ist schön, wenn es deutlich erkennbar den Stempel der bleibenden Persönlichkeit des Künstlers und des mehr oder weniger zufälligen Gefühls trägt, das der Anblick oder das Schauspiel, die von ihm wiedergegeben werden, bei ihm hervorgerufen haben. Mit anderen Worten, der Wert eines Kunstwerks liegt im Wesentlichen im Wert des 
Künstlers; es zieht uns an und ergreift uns aufgrund der Fähigkeiten und Eigenschaften des Künstlers, dessen Stempel es trägt. Je herausragender und ansprechender diese Fähigkeiten sind, desto mehr mögen und bewundern wir es. Aus demselben Grund verwerfen und verachten wir aber auch die kalten und banalen Kunstwerke, die durch diese Kälte und diese Banalität die geistige und moralische Mediokrität ihres Schöpfers zeigen und belegen, dass er seine Berufung verfehlt hat.

Folglich ist die Schönheit der Kunst eine rein menschliche Schöpfung, deren Mittel, wie in der Bildhauerei oder der Malerei, die Imitation sein kann, in der letztere jedoch ebenso gut nichts zu suchen haben kann, wie etwa in der Dichtung oder der Musik. Diese Schönheit ist von so besonderer Art, dass sie selbst im Hässlichen fortbesteht, denn die genaue Nachbildung einer hässlichen Figur kann durch die Gesamtheit der in ihrer Gestaltung aufgezeigten positiven Eigenschaften des Schöpfers ein schönes Kunstwerk sein.

Die Theorie der Nachahmung selbst ist nur eine unvollständige und oberflächliche Formel dessen, was wir hier vorstellen. Was bewundern wir an der Nachahmung? Die Ähnlichkeit? Sie ist im Gegenstand selbst sehr viel vollkommener. Aber wie kann die Ähnlichkeit mit einem hässlichen Gegenstand schön sein? Zwischen dem Gegenstand und dem Abbild muss natürlich ein neues Element hinzugekommen sein. Dieses Element ist die Persönlichkeit, ist zumindest die Geschicklichkeit des Künstlers. Und tatsächlich bewundern diejenigen, die behaupten, das Schöne bestehe in der Nachahmung, genau dies. Auch sie applaudieren letzten Endes dem Talent des Künstlers. Mag man noch so tief bohren, analysieren, auf dem Grund dieser Bewunderung wird man nichts anderes finden: Ob man will oder nicht, was man an einem Werk preist, ist der das Werk Schaffende.

Das war die Ansicht von Bürger, der in seinem Salon von 1863 schrieb: »In den Werken, die sie [die Öffentlichkeit] interessieren, setzen sich die Schöpfer in gewisser Weise an die Stelle der Natur. Wie vulgär sie auch sein mag, sie haben doch eine besondere und seltene Wahrnehmung von ihr. In dem Glas, das er gemalt hat, bewundern wir Chardin. In dem tiefgründigen und eigenartigen Charakter, den Rembrandt diesem banalen, vor ihm posierenden Kopf verliehen hat, bewundern wir Rembrandts Genie. Sieh an! So haben sie das gesehen! Und wie einfach oder wie seltsam in Ausdruck und Ausführung das doch ist! «

Ich glaube nicht, dass es nach diesen Ausführungen noch notwendig ist, uns damit aufzuhalten, die Theorie zu widerlegen, die das Kunstschöne auf die Interpretation der "schönen Natur « gründet. Trotz der glänzenden Bestätigung, die ihr drei Akademien gegeben haben, indem sie das Buch von Herrn Ch. Lévêque über La Science du Beau mit ihren jeweiligen Preisen ausgezeichnet haben, scheint sie uns auf keiner seriösen Grundlage zu beruhen, solange man uns nicht gezeigt hat, welches die »schöne Natur" ist, die in Junger Bettler, in Napoleon auf demSchlachtfeld von Preußisch-Eylau, in Das Floß der Medusa, in den Charakteren des Tartuffe oder der Marneffe interpretiert wird.

Schön im Kunstwerk ist nur, was der Künstler darin hineinlegt. Es ist das eigentliche Ergebnis seiner Anstrengung und die Bestätigung seines Erfolgs. Wann immer ein von einem beliebigen - körperlichen, seelischen oder geistigen - Eindruck zutiefst getroffener Künstler diesen Eindruck mithilfe eines beliebigen Verfahrens - Gedicht, Musik, Statue, Gemälde, Gebäude - so zum Ausdruck bringt, dass er in die Seele des Betrachters oder des Hörers dringt, ist das Kunstwerk schön, und zwar nach Maßgabe 
der Intelligenz, die es voraussetzt, der Tiefe des Eindrucks, den es ausdrückt, und der Ansteckungskraft, die ihm vermittelt wird.

Das Zusammentreffen dieser Bedingungen bildet den vollständigsten Ausdruck des Schönen.

Unter diesen Vorbehalten kann man zur Not die übliche Definition der Ästhetik beibehalten: die Wissenschaft vom Schönen. Für größere Klarheit und um jedwede Verwirrung zu vermeiden, möchten wir sie jedoch lieber die Wissenschaft vom Schönen in der Kunst nennen.

Gäbe es nicht die Tyrannei der vom Sprachgebrauch übernommenen Formeln, hätten wir sogar den Ausdruck »schön« gern ganz beiseitegelassen, hat er doch den Nachteil, sich zu ausschließlich auf den Sehsinn zu beziehen und an die Idee der sichtbaren Formen zu erinnern. Man versteht zur Not den Gebrauch dieses Wortes zu jener Zeit, als die Bildhauerei die Kunst schlechthin war. Um es auf die anderen Künste anzuwenden, musste man an ihm eine Reihe von Erweiterungen vornehmen, die ihn jeder Klarheit beraubten. Es gibt in der Sprache keinen verschwommeneren oder ungenaueren Ausdruck; die fehlende Präzision hat vielleicht mehr als man denkt zur Verwirrung der Ideen beigetragen, mit der allein die Vielzahl und die Absonderlichkeit der ästhetischen Theorien erklärt werden kann.

Man würde alle diese Nachteile und alle diese Unklarheiten vermeiden, sagte man einfach: Die Ästhetik ist die Wissenschaft, die die philosophische Untersuchung der Äußerungen des künstlerischen Genies zum Gegenstand hat.

\section{NOTES}

1. Und nicht der Glanz des Wahren, ein Ausdruck, den diejenigen Platon zuschreiben, die ihm die Phantasien ihrer eigenen Einbildungskraft unterschieben. Das Schöne, so wie der Mensch es begreifen kann, ist in seiner Lehre nur ein sehr trübes Abbild der göttlichen Vollkommenheit.

2. Aristoteles: Poetik, in: ders.: Hauptwerke, übersetzt von Wilhelm Nestle, Stuttgart, Alfred Kröner Verlag, S. 341. [A.d.Ü.]

3. Blaise Pascal: Gedanken, nach der endgültigen Ausgabe übertragen von Wolfgang Rüttenauer, Bremen, Carl Schünemann Verlag, 6. Aufl. 1964, S. 341. [A.d.Ü.]

4. In der ersten Auflage hatte Véron geschrieben: »Moses«. (A. d. Hg.)

5. Man kann von jedem Kunstwerk sagen, dass die Persönlichkeit des Künstlers dessen wichtigsten Wert ausmacht. Aber auf Ruisdael trifft das vielleicht noch mehr zu als auf jeden anderen. E. Fromentin, der die flämischen und holländischen Maler an Ort und Stelle mit wahrhaft bewundernswerter Sorgfalt und Scharfsinn untersucht hat, stellt fest, dass Ruisdael hinter vielen seiner Landsleute zurückbleibt, wenn man ihn vom Detail her untersucht. Es fehlt $\mathrm{ihm}$ an Kunstfertigkeit in einer Zeit und in einem Genre, wo alle fingerfertig waren. Er kann nicht gerade als gewandt bezeichnet werden. Er scheint einen schwerfälligen Geist zu haben, ohne Hintersinnigkeit, Beweglichkeit und Schalk. Seine Zeichenkunst hat nicht immer das Bissige, die Schärfe, das bizarr Getönte - Merkmale, die einigen Bildern Hobbemas eigen sind. Es ist ihm nie gelungen, eine Figur in seine Bilder zu setzen. Er hat nicht die goldene Atmosphäre von Cuyp; in der Plastizität ist er Terburg und Metzu unterlegen. Es fehlt ihm an Subtilität, geistiger 
Durchdringung, und die geistigen Finessen seiner Nacheiferer lassen ihn etwas farblos erscheinen. Seine Bilder ähneln sich, und wenn man viele davon hintereinander sieht, erscheinen sie am Ende eintönig. Seinen Bildern fehlt es an Farbvielfalt und Farbreichtum. Sie haben wenig Glanz, seine Farben sind nicht immer angenehm, und ihre Grundsubstanz ist nicht immer hervorragend. »Bei alledem ist Ruisdael dennoch einzigartig; man kann sich davon leicht im Louvre überzeugen, nach seinem Buisson, le Coup de soleil, la Tempête, le Petit Paysage $\left(\mathrm{n}^{\circ} 474\right)$ [...]. Bei der retrospektiven Ausstellung zugunsten der Elsass-Lothringer kann man sagen, dass Ruisdael dort mit einer offensichtlichen Souveränität herrschte, obwohl es in der Ausstellung außerordentlich viele holländische und flämische Meister gab [...]. Daran möchte ich all die erinnern, für die diese Ausstellung ausgezeichneter Werke ein Lichtstrahl war. Erwies Ruisdael sich dort nicht wie ein Meister und, noch achtbarer, wie ein großer Geist? In Brüssel, in Antwerpen, in Den Haag, in Amsterdam ist die Wirkung dieselbe. Wo immer Ruisdael erscheint, hat er eine ihm eigene Art aufzutreten, sich $\mathrm{zu}$ behaupten, Achtung $\mathrm{zu}$ gewinnen, Aufmerksamkeit $\mathrm{zu}$ erzeugen, die einem anzeigt, dass man die Seele einer bedeutenden Persönlichkeit vor sich hat, dass diese bedeutende Persönlichkeit von vornehmer Rasse ist und einem immer etwas Wichtiges $\mathrm{zu}$ sagen hat. Das ist er einzige Grund von Ruisdaels Überlegenheit, und dieser Grund genügt: Es gibt im Maler einen Menschen, der denkt, und in jedem seiner Werke eine Konzeption. In seinem Genre ebenso gebildet wie sein gebildetster Landsmann, ebenso natürlich begabt, überlegter und bewegter, besser als irgendein anderer hat er seinen Talenten ein Gleichgewicht hinzugefügt, das die Einheit des Werkes und die Vollkommenheit der Werke hervorbringt. Sie werden in seinen Bildern einen Hauch von Vollendung, von Sicherheit, von tiefem Frieden wahrnehmen, der das Unterscheidungsmerkmal seiner Person ist und der beweist, dass zwischen seinen angeborenen Vermögen, seiner großen Erfahrung, seiner immer lebhaften Sensibilität, seiner immer gegenwärtigen Reflexion ein ständiger Einklang bestand. Ruisdael malt wie er denkt, mit gesundem Verstand, stark, weit.« Man kann den Einfluss des Menschen auf das Werk nicht besser vor Augen führen, was ja gerade die These ist, die wir vertreten, und der einzige und solide Grund der Ästhetik, so wie wir sie verstehen. [Eugène Fromentin, Les maîtres d'autrefois (1876), VII; éd. Garnier frères, Paris, 1972, S. 159 ff. (Anm. d. Hg.)]

\section{INDEX}

Schlüsselwörter : Ästhetik, das Schöne, Nachahmungstheorie

Mots-clés : esthétique, le beau, théorie de l'imitation

\section{AUTEURS \\ EUGÈNE VÉRON}

Historiker und Philosoph. (Nähere Informationen $\underline{\text { hier) }}$ 